\title{
A Comparative Magnetic Resonance Imaging Study of the Anatomy, Variability, and Asymmetry of Broca's Area in the Human and Chimpanzee Brain
}

\author{
Simon S. Keller, ${ }^{1,2}$ Neil Roberts, ${ }^{3}$ and William Hopkins ${ }^{4,5}$ \\ ${ }^{1}$ Department of Neurology, University of Münster, D-48149 Münster, Germany, ${ }^{2}$ The Magnetic Resonance and Image Analysis Research Centre, University \\ of Liverpool, Liverpool L69 3GE, United Kingdom, ${ }^{3}$ Division of Medical and Radiological Sciences, University of Edinburgh, Edinburgh EH16 4TJ, United \\ Kingdom, ${ }^{4}$ Division of Psychobiology, Yerkes National Primate Research Center, Emory University, Atlanta, Georgia 30322, and ${ }^{5}$ Department of \\ Psychology, Agnes Scott College, Decatur, Georgia 30030
}

The frontal operculum - classically considered to be Broca's area- has special significance and interest in clinical, cognitive, and comparative neuroscience given its role in spoken language and the long-held assumption that structural asymmetry of this region of cortex may be related to functional lateralization of human language. We performed a detailed morphological and morphometric analysis of this area of the brain in humans and chimpanzees using identical image acquisition parameters, image analysis techniques, and consistent anatomical boundaries in both species. We report great inter-individual variability of the sulcal contours defining the operculum in both species, particularly discontinuity of the inferior frontal sulcus in humans and bifurcation of the inferior precentral sulcus in chimpanzees. There was no evidence of population-based asymmetry of the frontal opercular gray matter in humans or chimpanzees. The diagonal sulcus was only identified in humans, and its presence was significantly $(F=12.782, p<0.001)$ associated with total volume of the ipsilateral operculum. The findings presented here suggest that there is no population-based interhemispheric macroscopic asymmetry of Broca's area in humans or Broca's area homolog in chimpanzees. However, given that previous studies have reported asymmetry in the cytoarchitectonic fields considered to represent Broca's area-which is important given that cytoarchitectonic boundaries are more closely related to the regional functional properties of cortex relative to sulcal landmarks-it may be that the gross morphology of the frontal operculum is not a reliable indicator of Broca's area per se.

\section{Introduction}

The study of interhemispheric cerebral asymmetries in nonhuman primates represents an important scientific endeavor given that hemispheric specialization, which may be mediated by such asymmetries, is suggested to be related to language (Witelson and Kigar, 1988; Corballis, 2003; Annett, 2006). Therefore, demonstration of human-like neuroanatomical asymmetries in closely related phylogenetic species such as the great apes may indicate a potential substrate for the development of advanced cognition in these species and offer insights into the organization of human cortical language areas (Gannon et al., 1998). There has been a recent proliferation of magnetic resonance imaging (MRI) studies of cerebral asymmetry in the great ape brain, which have provided evidence to suggest that human cerebral asymmetries

Received June 18, 2009; revised July 30, 2009; accepted Aug. 29, 2009.

S.S.K. was supported by a European Commission Grant from the Sixth Framework Programme entitled "Paul Broca II: The Evolution of Cerebral Asymmetry in Homo Sapiens" Project 12880. W.H. was supported by National Institutes of Health Grants NS-42867, HD-38105, and HD-56232. The acquisition of human MRI scans was funded by the T. J. Crow Psychosis Trust. We thank Valarie Adams at the Magnetic Resonance and Image Analysis Research Centre for her assistance in the application of the human MRI protocol and all human volunteers for their participation.

Correspondence should be addressed to Dr. Simon Keller, Department of Neurology, University of Münster, Albert-Schweitzer Straße 33, 48149 Münster, Germany. E-mail: s.s.keller@uni-muenster.de.

DOI:10.1523/JNEUROSCI.2892-09.2009

Copyright $\odot 2009$ Society for Neuroscience ～0270-6474/09/2914607-10\$15.00/0 postulated to be important for hemispheric specialization of language are observed in great apes, including fronto-occipital torque (Hopkins and Marino, 2000; Pilcher et al., 2001; Hopkins et al., 2008) and leftward morphological asymmetry of the planum temporale (Gannon et al., 1998; Hopkins et al., 1998) and frontal operculum (Cantalupo and Hopkins, 2001). To build on these studies, we performed an MRI investigation to compare cerebral asymmetry between great apes and humans within the same study using the same image acquisition protocol, anatomical definitions, and quantitative MR image analysis techniques. In particular, we compared the morphology, morphological variability, and volume asymmetry of the frontal operculum between the human and chimpanzee brain.

The frontal operculum-frequently referred to as Broca's area-located in the posterior inferior frontal gyrus in humans, has generated special interest for the search of neuroanatomical asymmetries given its role in language. Keller et al. (2009b) have recently shown that the literature indicates that morphological asymmetry of the frontal operculum may exist in the human brain, but such asymmetry is not a robust finding and may be dependent on methodological approaches. Although it has been shown that asymmetry of the frontal operculum may be consistent with language lateralization in some cases (Foundas et al., 1996; Dorsaint-Pierre et al., 2006), predicting language lateralization in humans by virtue of interhemispheric asymmetries of this region is seemingly not possible (Keller et al., 2007b, 2009b). 
There were three primary objectives of the present study. First, we sought to describe, quantify, and compare the anatomy and morphological variability of the frontal operculum in humans and chimpanzees. Second, using homologous anatomical boundaries and three-dimensional sampling of the gray matter using the Cavalieri method of design-based stereology, we sought to determine volumetric asymmetries of the frontal operculum and compare population-based directional asymmetry between humans and chimpanzees. Finally, we sought to investigate the sulcal configurations affecting volumetric asymmetry. These objectives were sought to be achieved using an equal number of chimpanzee and human MR images acquired using the same acquisition protocol on the same type of MR scanner, with measurements obtained using identical MR image analysis techniques.

\section{Materials and Methods}

Subjects and MR image acquisition. We obtained three-dimensional T1-weighted magnetizationprepared rapid-acquisition gradient echo (MPRAGE) MR images for 30 chimpanzees and 30 humans using a Siemens 3 tesla Trio MR system. Chimpanzee images were acquired at Yerkes National Primate Research Centre (YNPRC) in Atlanta, Georgia. Human images were acquired at the Magnetic Resonance and Image Analysis Research Centre at the University of Liverpool (Liverpool, UK). The MR sequences used for acquisition of images are shown in Table 1. Acquisition parameters were made to be as similar as possible for humans and chimpanzees. The voxel resolution was slightly reduced in the $y$ direction in humans. Reducing the voxel resolution to $1.0 \mathrm{~mm}$ in the $y$ direction in humans resulted in an acquisition time of $12 \mathrm{~min}$, which contrasted to the 36 $\min (y=0.6 \mathrm{~mm})$ chimpanzee scan. However, the in-plane voxel resolution was $0.6 \times 0.6 \mathrm{~mm}$ for both humans and chimpanzees, resulting in a very similar between-tissue contrast (Fig. 1). All humans provided written consent to participate in this study, which had local ethics committee approval. For the chimpanzee scans, subjects were first immobilized by ketamine injection $(10 \mathrm{mg} / \mathrm{kg})$ and subsequently anesthetized with propofol (40-60 $\mathrm{mg} \cdot \mathrm{kg}^{-1} \cdot \mathrm{h}^{-1}$ ) following standard procedures at the YNPRC. Subjects were then transported to the MRI facility and remained anesthetized for the duration of the scans as well as the time needed to transport them between their home cage and the imaging facility (total time, $\sim 2 \mathrm{~h}$ ). Subjects were placed in the scanner in a supine position with their head fitted inside the human-head coil.

Anatomical definitions. The volume of the frontal operculum was estimated in the left and right cerebral hemispheres for all chimpanzees and humans. Strictly speaking, the term "frontal operculum" is anatomically incorrect in the great ape, because this region of the inferior frontal gyrus does not form an operculum over the insula in any of the great ape species. Taking the precedent from the studies in humans, however, we refer to the region of the inferior frontal gyrus measured in the present study as the frontal operculum in humans and chimpanzees. Figure 2 schematically illustrates this region of cortex in the human and chimpanzee brain, and Figure 3 indicates this region from the surface of the brain in a randomly selected human and chimpanzee studied in the present investigation.

We have described previously in detail the anatomy and sulcal variability defining the frontal operculum in humans (Keller et al., 2007a,
Table 1. MRI acquisition information for the study images

\begin{tabular}{lll}
\hline & Chimpanzee & Human \\
\hline$n$ & 30 & 30 \\
Male-female & $14-16$ & $13-17$ \\
MRI scanner & 3T Siemens Trio, CP Head Coil & 3T Siemens Trio, CP Head Coil \\
Sequence & MPRAGE T1-weighted & MPRAGE T1-weighted \\
TR & $2300 \mathrm{~ms}$ & $2300 \mathrm{~ms}$ \\
TE & $4.4 \mathrm{~ms}$ & $4.4 \mathrm{~ms}$ \\
TI & $1100 \mathrm{~ms}$ & $1100 \mathrm{~ms}$ \\
Flip angle & 8 & 8 \\
NEX & 3 & 1 \\
FOV & $200 \times 200 \mathrm{~mm}$ & $200 \times 200 \mathrm{~mm}$ \\
In-plane resolution & $0.625 \times 0.625 \mathrm{~mm}$ & $0.625 \times 0.625 \mathrm{~mm}$ \\
Slice thickness & $0.6 \mathrm{~mm}$ & $1.0 \mathrm{~mm}$ \\
Data matrix size & $320 \times 320$ & $320 \times 320$ \\
Slices & 192 & 192 \\
Acquisition time & $36 \mathrm{~min}$ & $12 \mathrm{~min}$ \\
\hline
\end{tabular}

TR, Repetition time; TE, echo time; IT, inversion time; NEX, number of excitations; FOV, field of view.

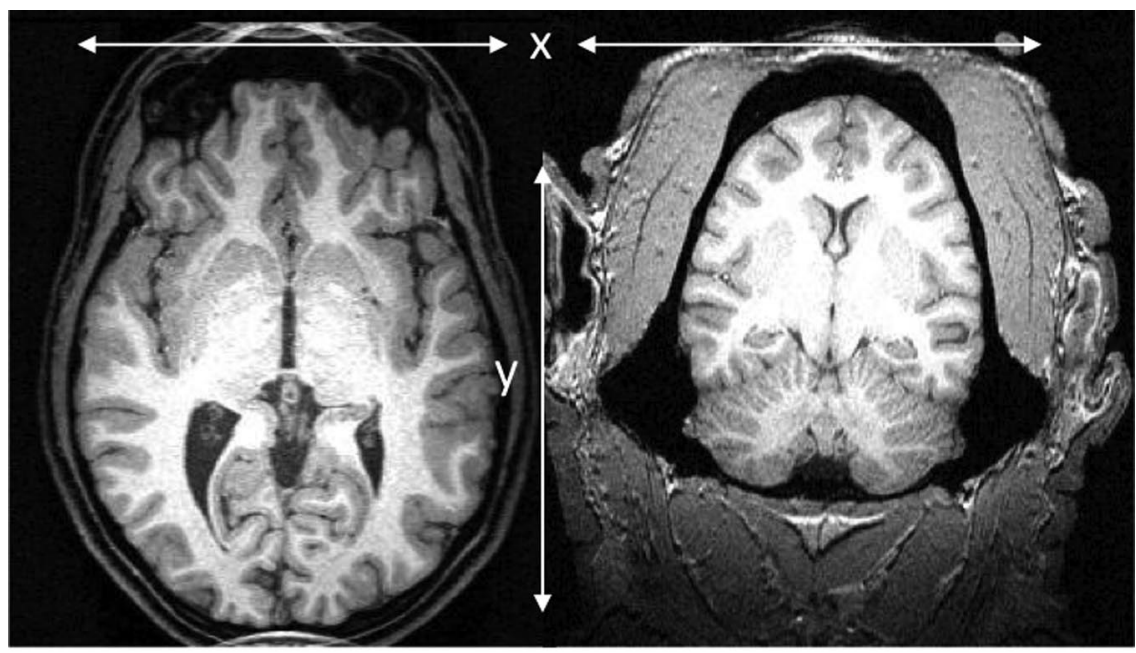

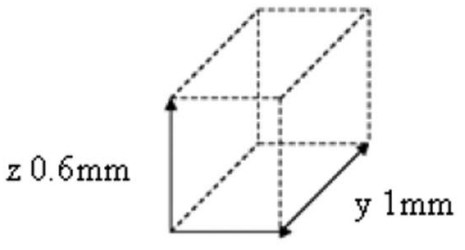

$\mathrm{x} 0.6 \mathrm{~mm}$

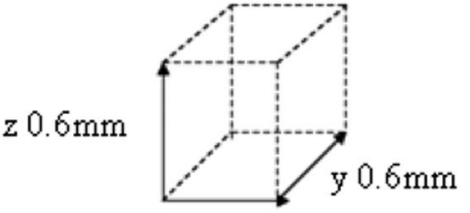

$\mathrm{x} 0.6 \mathrm{~mm}$
Figure 1. An axial section of the human (left) and chimpanzee (right) T1-weighted MPRAGE sequence acquired in the present study. The voxel sizes are schematically illustrated beneath the image.

2009b). The inferior frontal gyrus can be divided into three subregions by virtue of the ascending and horizontal rami of the Sylvian fissure, the inferior frontal sulcus and the inferior precentral sulcus. The major rami divide the inferior frontal gyrus into a posterior third (pars opercularis), anterior third (pars triangularis), and ventral third (pars orbitalis), of which the pars triangularis is ordinarily larger than the other constituent regions. The pars opercularis-referred to as the frontal operculum in this study-is demarcated caudally from the ventral precentral gyrus by the inferior precentral sulcus, dorsally from the middle frontal gyrus by the inferior frontal sulcus, and rostrally from the pars triangularis by the anterior ascending ramus of the Sylvian fissure (Figs. 2, 3). Within the human frontal operculum, there is occasionally the diagonal sulcus (Figs. 2, 3 ), although the term "diagonal" maybe somewhat misleading given that this sulcus is rarely diagonal per se and does not have a uniform appearance. There is great inter-individual variability in the shape, length, continuity, and number of these sulcal contours, which gives rise to the great variability in size, surface area, and volume of the frontal operculum (Ono et al., 1990; 


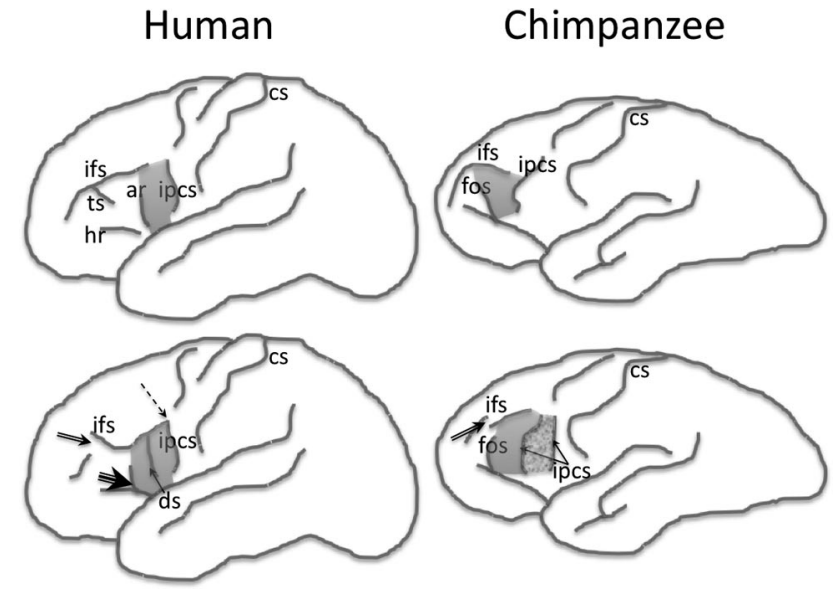
human (left) and chimpanzee (right) brain (not to scale). The top row indicates the typical sulcal configurations, and the bottom row indicates the variability of the sulcal contours based on the data reported by Keller et al. (2007b) in humans and Sherwood et al. (2003) in chimpanzees. Arrows on the bottom row indicate the "deviation" from the typical configuration: single arrow, presence of a new sulcus (ds/ipcs); double arrow, discontinuity of a sulcus (ifs); triple arrow, presence of connection of rami (ar/hr); dashed arrow, presence of connection of sulci (ipcs/ifs). The shaded area indicates the frontal operculum in both species. The grainy shaded area in the bottom right illustration indicates the possible extension of the operculum when an additional segment of the inferior precentral sulcus is present. ar, Anterior ascending ramus of the Sylvian fissure; cs, central sulcus; ds, diagonal sulcus; fos, fronto-orbital sulcus; ifs, inferior frontal sulcus; ipcs, inferior precentral sulcus.
Figure 2. Schematicillustrations of the sulcal contours defining the frontal operculum in the

chimpanzee frontal operculum forms the posterior portion of the inferior frontal gyrus, which is delineated from the precentral gyrus caudally by the inferior precentral sulcus and from the middle frontal gyrus dorsally by the inferior frontal sulcus (Cantalupo and Hopkins, 2001). However, the anterior aspect of the frontal operculum in chimpanzees is demarcated from the remainder of the inferior frontal gyrus by the fronto-orbital sulcus and not the anterior ascending ramus as in humans (Fig. 2, 3). The ventralmost point for measurements was where the inferior precentral sulcus terminates, and the dorsalmost point was, like humans, the fundus of the inferior frontal sulcus. As in humans, the region of interest in the present study encompassed all gray matter of the frontal operculum, excluding white matter.

MR image analysis. All human and chimpanzee images were skul stripped, cerebral hemispheres were extracted (segmented from brainstem and cerebellum), and the cortical surface was reconstructed using the previously validated automated algorithms of Freesurfer software (http://surfer.nmr.mgh.harvard.edu/). This step enabled the investigator to view the sulcal contours from the external convexity of the brain.

Before volume estimation, the human images were interpolated from anisotropic (acquired voxel size of $0.6 \mathrm{~mm} / 0.6 \mathrm{~mm} / 1 \mathrm{~mm}$ ) into isotropic $(0.6 \mathrm{~mm} / 0.6 \mathrm{~mm} / 0.6 \mathrm{~mm})$ using MRIcro software (http://www.sph. sc.edu/comd/rorden/mricro.html). No image interpolation was performed for the chimpanzees given that images were already isotropic (see acquisition details). Volume estimation of the frontal operculum in al humans and chimpanzees was performed using MEASURE software (Barta et al., 1997). MEASURE permits stereological volume estimation using point counting techniques in orthogonal sagittal, coronal, and axial planes simultaneously. We have previously applied stereological techniques to estimate the volume of the frontal operculum in humans using other software packages that permit quantification in only the coronal plane (Keller et al. 2007a,b; Lux et al., 2008). In these previous studies, before volume estimation, images were imported into Brainvoyager software (http:// brainvoyager.com) to allow the investigator to manually place markers in the three orthogonal planes to guide point counting in the coronal plane. The use of MEASURE removed the need to place markers as sampling of the entire frontal operculum could be performed in three dimensions (Keller and Roberts, 2009).

The previously described (Keller et al., 2007a) stereological parameters for measurement of the frontal operculum were again used in MEASURE software (sampling density of 3 pixels, $0.186 \mathrm{~cm}$ ). To achieve a coefficient of error of less than or equal to $5 \%$ (Roberts et al. 2000) while maximizing time efficiency, we found that $\sim 200-300$ points per structure should be counted (Roberts et al., 2000; Keller et al., 2002a,b, 2007a, 2009a; Keller and Roberts, 2009). Both human and chimpanzee stereological parameters were optimized based on this approach. Given that the chimpanzee brain is smaller than that of a human, we defined the stereological parameters for measurement of the frontal operculum in chimpanzees as 2 pixels $(0.125 \mathrm{~cm})$. It is important to note that the property of unbiasedness of the vol-

Tomaiuolo et al., 1999; Keller et al., 2007a). We have previously discussed the factors that can affect volume estimation of the frontal operculum, which include the presence of discontinuous sulci, bifid sulci, absent rami, misclassification of sulci, and submerged gyri (Keller et al., 2009b). As in our previous studies (Keller et al., 2007a,b; Lux et al., 2008), we sampled the entire gray matter of the frontal operculum, from the fundus of the inferior frontal sulcus dorsally to the fundus of the circular insular sulcus ventrally, including all lateral cortex.

The anatomical definitions of the frontal operculum in the chimpanzee were as similar as possible to human definitions. As in humans, the ume estimate is not affected by the sampling intensity. Using the parameters $2 \times 2 \times 2$ and $4 \times 4 \times 4$ will both yield unbiased volume estimates for the frontal operculum, but the coefficient of error will be lower (i.e., the precision will be higher) for the former.

Examples of point counting for volume estimation of the frontal operculum in human and chimpanzee brains are presented in Figures 4 and 5, respectively. No single plane was preferentially used during point counting. However, particular views did permit optimal identification of some sulcal boundary structures. For example, coronal sections best identify 
deep posterior regions of the frontal operculum that are submerged beneath the precentral gyrus, sagittal sections best identify the anterior limits of the frontal operculum determined by the anterior ascending ramus (humans) and fronto-orbital sulcus (chimpanzees), and axial sections best identify the full intrasulcal anatomy of inferior sections of the frontal operculum. When a point was marked (using the mouse button) in one plane as being in the region of interest, the same point on the orthogonal sections was marked (yellow). Marked points could be "unmarked," again with a click of the mouse button. All marked points were visualized in all three orthogonal planes to confirm that only the gray matter within the frontal operculum was sampled.

\section{Results}

\section{Repeatability and}

reproducibility analyses

Intra-rater and inter-rater reliability for volume estimation of the human frontal operculum using stereological techniques has been demonstrated previously (Keller et al., 2007a). To ensure repeatability and reproducibility of volume estimates for the chimpanzee frontal operculum, intra-rater and inter-rater studies were administered on 10 randomly selected chimpanzee scans from the study cohort. For the intrarater study, measurements were compared between two measurements of the opercula by the same investigator at least 2 weeks apart. For the inter-rater analysis, measurements were contrasted between two investigators. Results indicated excellent levels of intra-rater (intraclass correlation of 0.99 ) and inter-rater (intraclass correlation of 0.96 ) reliability for measurements of the chimpanzee frontal operculum.

\section{Anatomical variability}

Results for all individual subjects are provided in the supplemental data (available at www.jneurosci.org as supplemental material).

\section{Human}

Results from visual assessment of the variability of the sulcal contours defining the frontal operculum in the human brain are presented in Table 2. There was morphological variability of the sulcal contours, particularly of the inferior frontal sulcus, which was discontinuous and consisted of two segments in $40 \%$ of human hemispheres. Furthermore, more than one ventral segment of the inferior precentral sulcus was identified in 5\% of hemispheres, and the diagonal sulcus was identified in $76.7 \%$ of hemispheres. The diagonal sulcus did not constitute a uniform appearance in all humans, was of various lengths and depths, and was differentially connected to local sulci. Of the 46 diagonal sulci observed in the present study, 18 had no connection with surrounding sulci, and 28 connected to either one or a combination of the inferior precentral sulcus, inferior frontal sulcus, or anterior ascending ramus (see supplemental material, available at www.jneurosci.org). Figure 6 shows some examples of the diagonal sulci observed in the present study.

\section{Chimpanzee}

Results from visual assessment of the variability of the sulcal contours defining the frontal operculum in the chimpanzee brain are presented in Table 3. There was morphological variability of the sulcal contours, particularly of the inferior precentral sulcus. This was found to consist of one individual segment in $76.7 \%$ of hemispheres, two segments in $20 \%$ of hemispheres, and three ventral segments in $3.3 \%$ of hemispheres. Figure 7 illustrates some cases of multiple segments of the inferior precentral sulcus observed in the present study. The inferior frontal sulcus was a continuous single sulcus in $83.3 \%$ of hemispheres. In these cases, the sulcus usually connected with the inferior precentral sulcus and extended anteriorly until fronto-polar regions. The inferior frontal sulcus was discontinuous in $16.7 \%$ of hemispheres and always consisted of two segments. An example of a discontinuous chimpanzee inferior frontal sulcus is provided in Figure 8. The frontoorbital sulcus was present in all hemispheres studied and did not 


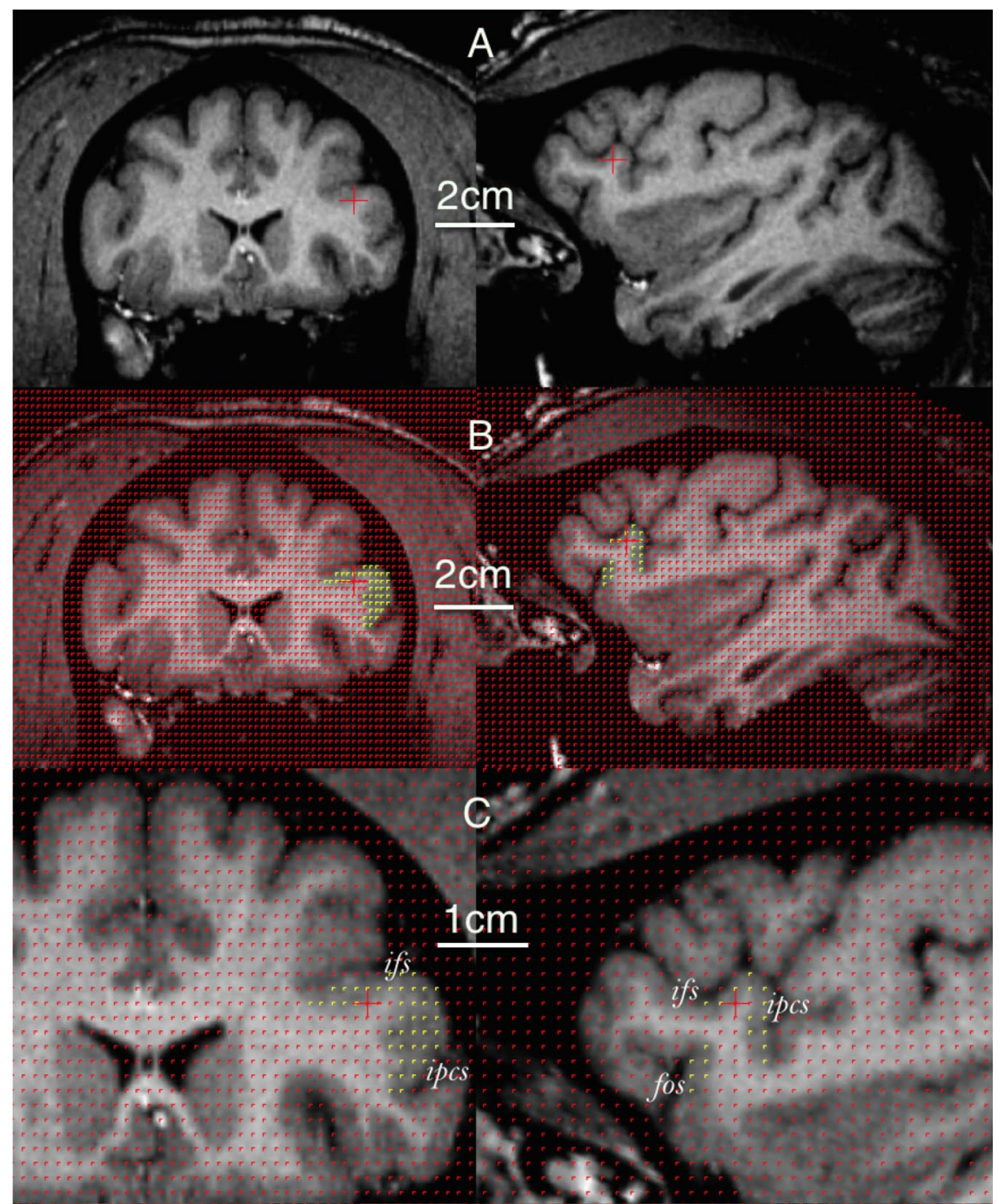

Figure 5. Volume estimation of the homologous frontal operculum in a chimpanzee brain using stereology. Format as in Figure 4. fos, Fronto-orbital sulcus; ifs, inferior frontal sulcus; ipcs, inferior precentral sulcus.

Table 2. Morphological variability in humans

\begin{tabular}{llllc}
\hline Structure & Morphology & Left hemisphere & Right hemisphere & Total \\
\hline ipcs & One segment & $100 \%(30)$ & $(90 \%) 27$ & $95 \%(57)$ \\
& Two segments & 0 & $10 \%(3)$ & $5 \%(3)$ \\
& Three segments & 0 & 0 & 0 \\
ifs & One segment & $56.7 \%(17)$ & $63.3 \%(19)$ & $60 \%(36)$ \\
& Two segments & $43.3 \%(13)$ & $36.6 \%(11)$ & $40 \%(24)$ \\
& Three segments & 0 & 0 & 0 \\
ar & Vertical & $83.3 \%(25)$ & $80 \%(24)$ & $81.7 \%(49)$ \\
& Oblique & $6.7 \%(2)$ & $10 \%(3)$ & $8.3 \%(5)$ \\
& Diagonal & $(10 \%) 3$ & $10 \%(3)$ & $10 \%(6)$ \\
ds & Not present & $30 \%(9)$ & $16.7 \%(5)$ & $23.3 \%(14)$ \\
& No connection & $26.7 \%(8)$ & $33.3 \%(10)$ & $30 \%(18)$ \\
& Connection & $43.3 \%(13)$ & $50 \%(15)$ & $46.7 \%(28)$ \\
& &
\end{tabular}

ar, Anterior ascending ramus of the Sylvian fissure; $d s$, diagonal sulcus; ifs, inferior frontal sulcus; ipcs, inferior precentra sulcus. An oblique as is one that is neither vertical or diagonal and tends to curl in different directions through parasagittal sections. Some diagonal armay become morevertical medially (supplemental Appendix, available at www.jneurosci.org as supplemental material). The ds may be connected to one or a combination of thear, ifs, or ipcs. For an individual breakdown of values, see supplemental material (available at www.jneurosci.org)

connect with any surrounding sulci defining the frontal operculum. It was a single deep sulcus in $95 \%$ of hemispheres. The remaining fronto-orbital sulci consisted of two (3.33\%) or three $(1.66 \%)$ branches. In no case was an additional sulcus present within the frontal operculum (i.e., a homolog to the diagonal sulcus in humans).

\section{Volume and asymmetry}

The mean volume for the left and right frontal operculum in humans was 4.96 $\mathrm{cm}^{3}$ (minimum, $2.51 \mathrm{~cm}^{3}$; maximum, $9.48 \mathrm{~cm}^{3}$; SD, 1.73) and $5.00 \mathrm{~cm}^{3}$ (minimum, $1.85 \mathrm{~cm}^{3}$; maximum, $8.27 \mathrm{~cm}^{3}$; SD, 1.66), respectively. This interhemispheric difference was not significantly different (df $=29, t=-0.116, p=0.908$ ). Fourteen of the 30 humans had leftward asymmetry of the frontal operculum. The remaining 16 humans had rightward volume asymmetry. When the anterior segment of the inferior precentral sulcus was taken in chimpanzees with more than one segment, the mean volume of the left and right frontal operculum was $1.12 \mathrm{~cm}^{3}$ (minimum, $0.50 \mathrm{~cm}^{3}$; maximum, 1.75 $\mathrm{cm}^{3}$; $\mathrm{SD}, 0.35$ ) and $1.17 \mathrm{~cm}^{3}$ (minimum, $0.68 \mathrm{~cm}^{3}$; maximum, $2.03 \mathrm{~cm}^{3}$; SD, 0.34 ), respectively. This interhemispheric difference was not statistically significant $(\mathrm{df}=$ $29, t=-0.673, p=0.506)$. Twelve of the 30 chimpanzees had leftward asymmetry of the frontal operculum, whereas the remaining 18 chimpanzees had rightward asymmetries.

The variable morphology of the inferior precentral sulcus had an effect on volume estimation of the frontal operculum in all chimpanzee hemispheres with more than one segment, and, importantly, volume asymmetry changed direction based on the segment taken for boundary purposes. The volumes for all chimpanzees with more than one segment of the inferior precentral sulcus are presented in Table 4. There were two predominantly interesting findings; first, volume asymmetry was rightward in 9 of the 10 chimpanzees with more than one segment of the inferior precentral sulcus. In only one subject was there leftward volume asymmetry when this sulcus was bifurcated. Second, volume asymmetry of the frontal operculum changed direction in four subjects (three from rightward to leftward, one from leftward to rightward) when the posterior segment of the inferior precentral sulcus was taken. When the posterior segment was taken, the mean volume of the left and right frontal operculum was $1.28 \mathrm{~cm}^{3}$ (minimum, $0.83 \mathrm{~cm}^{3}$; maximum, $2.37 \mathrm{~cm}^{3}$; SD, 0.35) and $1.32 \mathrm{~cm}^{3}$ (minimum, 0.68 $\mathrm{cm}^{3}$; maximum, $2.51 \mathrm{~cm}^{3}$; SD, 0.51), respectively. This interhemispheric difference was not statistically significant ( $\mathrm{df}=29$, $t=-0.436, p=0.666)$.

A between-species comparison of the individual volume asymmetries and group mean volumes is presented in Figure 9. Interhemispheric volume asymmetries [calculated using: $(r-l) /$ $(r+l) \times 2$; negative values are leftward asymmetry and vice versa] are relatively consistent between groups, with both species showing evidence of a slight rightward groupwise asymmetry and both including more cases with rightward volume asymmetry. The mean asymmetry values for humans and chimpanzees 

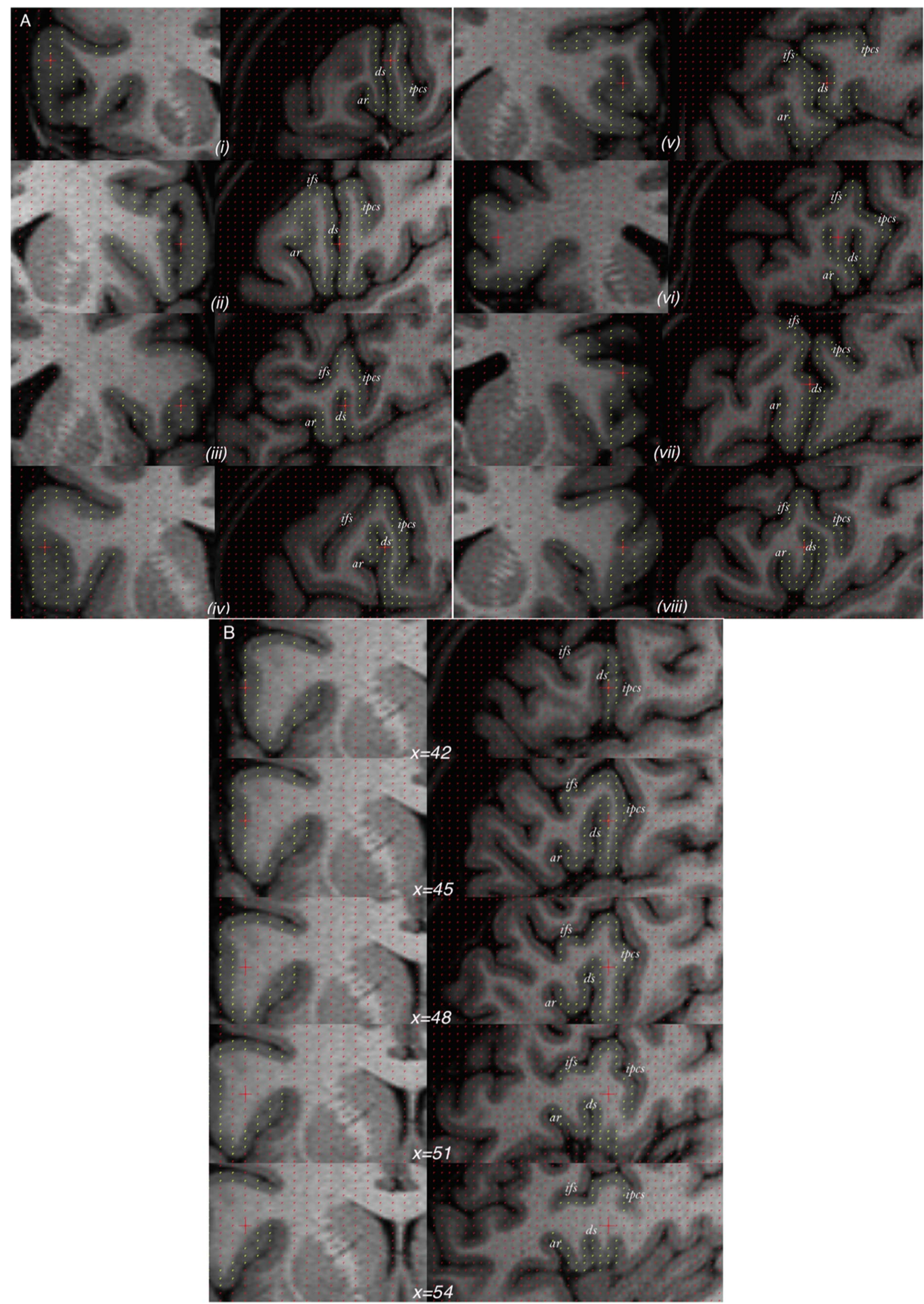

Figure 6. The variability of the diagonal sulcus in humans. $A$, Eight randomly chosen cases illustrated using orthogonal coronal (left) and sagittal (right) sections. $B$, Example of one case in which, from the surface of the brain, the anterior ascending ramus is barely visible and could bemistaken forthe diagonal sulcus. Only through navigation through theintrasulcal anatomy [indicated through thex (i.e., left-right) dimension] can the anterior ascending ramus be correctly identified. Yellow points mark the frontal operculum. ar, Anterior ascending ramus of the Sylvian fissure; ds, diagonal sulcus; ifs, inferior frontal sulcus; ipcs, inferior precentral sulcus. 
Table 3. Morphological variability in chimpanzees

\begin{tabular}{lllcc}
\hline Structure & Morphology & Left hemisphere & Right hemisphere & Total \\
\hline Ipcs & One segment & $73.3 \%(22)$ & $80 \%(24)$ & $76.7 \%(46)$ \\
& Two segments & $23.3 \%(7)$ & $16.7 \%(5)$ & $20 \%(12)$ \\
\multirow{4}{*}{ Ifs } & Three segments & $3.3 \%(1)$ & $3.3 \%(1)$ & $3.3 \%(2)$ \\
& One segment & $86.7 \%(26)$ & $80 \%(24)$ & $83.3 \%(50)$ \\
& Two segments & $13.3 \%(4)$ & $20 \%(6)$ & $16.7 \%(10)$ \\
\multirow{3}{*}{ Fos } & Three segments & 0 & 0 & 0 \\
& One branch & $93.3 \%(28)$ & $93.3 \%(28)$ & $93.3 \%(56)$ \\
& Two branches & $3.3 \%(1)$ & $6.7 \%(2)$ & $5 \%(3)$ \\
& Three branches & $3.3 \%(1)$ & 0 & $1.7 \%(1)$ \\
\hline
\end{tabular}

fos, Fronto-orbital sulcus; ifs, inferior frontal sulcus; ipcs, inferior precentral sulcus. (based on anterior inferior precentral sulcus measurements) were 0.01 (maximum leftward, - 1.07; maximum rightward, $0.80 ; \mathrm{SD}, 0.45$ ) and 0.05 (maximum leftward, -0.65 ; maximum rightward, $0.77 ; \mathrm{SD}, 0.36$ ), respectively. This was not significantly different $(\mathrm{df}=58, F=1.366, p=0.247)$.

When we analyzed the effect of morphological variability on volume, we found that the presence of a diagonal sulcus had a significant effect on the volume of the frontal operculum in the 60 human hemispheres [volume/no sulcus/sulcus with no connection/sulcus with connection] (ANOVA, $\mathrm{df}=58, F=12.782, p<$ $0.001)$. In particular, the smallest opercula were those without a diagonal sulcus, and the largest were generally the opercula with a diagonal sulcus that connected to the inferior precentral sulcus, inferior frontal sulcus, or anterior ascending ramus (Fig. 10). This effect was independent of laterality and existed regardless of the hemisphere side. The number of segments of the inferior frontal sulcus did not affect volume estimation of the human operculum [volume/one segment/two segments] $(\mathrm{df}=58$, $t=-0.001, p=0.99)$.

\section{Discussion}

There were three principle findings of the present study. First, we report that humans and chimpanzees demonstrate inter-individual and interhemispheric variability in the sulcal contours defining the frontal operculum. In particular, chimpanzees had an increased presence of multiple segments of the inferior precentral sulcus relative to humans, and humans had an increased presence of multiple segments of the inferior frontal sulcus. Second, we found that both humans and chimpanzees do not show evidence of population-based asymmetry of the gray matter of the frontal operculum. Third, the presence of the diagonal sulcus in humans significantly increased the volume of the frontal operculum, particularly when connected with surrounding sulci. However, given that there was no interhemispheric difference in the presence of the diagonal sulcus, there was no interhemispheric volume asymmetry of the operculum.

Schenker et al. (2009) examined neuron density and volume of area 44 and area 45 in a sample of 12 chimpanzees, and several findings from that study are germane to the present investigation. First, area 44 was principally found on the gyrus lying between the fronto-orbital and precentral inferior sulci, consistent with the original report by Bailey et al. (1950). In contrast, area 45 was found in cortex anterior to the fronto-orbital sulcus and was thus located in regions outside the gyrus quantified in this study. Second, for both area 44 and area 45 , asymmetries were not found in either the volume or neuron density. This observation differs from at least two reports in
Figure 7. Multiple segments of the inferior precentral sulcus in the chimpanzee brain. $\boldsymbol{A}$, Three ventral segments (indicated by "seg") in the right hemisphere of case 2. B, Two ventral segments in the left hemisphere of case 14. C, Two parallel segments in the right hemisphere of case 28. The two parallel segments are of the type schematically illustrated in Figure 2. 
humans (Amunts et al., 1999; Uylings et al., 2006), but the sample size was small in the chimpanzees and this is particularly problematic when variability in the size of a structure is so robust. Finally, in humans, handedness and other subject variables are likely to be more consistent, although this is not the case in the chimpanzees and this might explain some between species variability. For example, within this sample of 30 chimpanzees, 11 chimpanzees prefer to gesture with their left hand and 19 prefer to gesture with their right. Within the left-handed sample, 9 of the 11 subjects (82\%) showed a

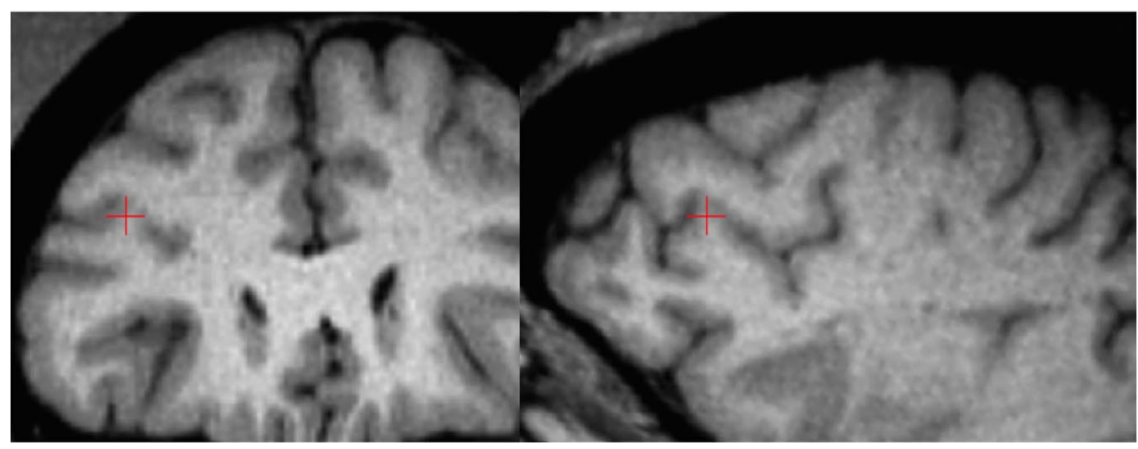

Figure 8. A discontinuous left inferior frontal sulcus (ifs) in chimpanzee case 14 resulting in two segments of the ifs. This discontinuity is of the type schematically illustrated in Figure 2. right hemisphere asymmetry, whereas within the right-handed group, 9 of the 19 (47\%) had a right hemisphere bias. Although not significantly different, the trends clearly point to a potential modulating effect of handedness on this asymmetry, at least in the chimpanzees.

The diagonal sulcus was only observed in humans. Results obtained in the present study confirm our previous findings, indicating that the presence of a diagonal sulcus significantly increases the volume of the frontal operculum, and therefore the unilateral presence of this sulcus can predict volume asymmetry of the operculum in individual cases (Keller et al., 2007a). The presence of the diagonal sulcus increases both the cortical volume and presumably the cortical connectivity of the pars opercularis, given the increased intrasulcal surface area. Furthermore, we extend these findings by reporting that diagonal sulci with connections to local sulci and rami increase the volume of the frontal operculum relative to diagonal sulci with no connections. We did not observe a left-greater-than-right incidence of diagonal sulci, which is contrary to our previously reported finding (Keller et al., 2007a, 2009b) but consistent with the findings reported by Knaus et al. (2007). We were unable to identify a homologous sulcus between the inferior precentral sulcus, inferior frontal sulcus, and fronto-orbital sulcus in the chimpanzee brain. The definition of this sulcus is rarely discussed in the literature, and it is crucial to objectively identify this sulcus. In some human hemispheres without a diagonal sulcus in the present study, there was occasionally a small dimple that did not constitute a full sulcus. It has been reported previously that these dimples exist in almost all human frontal opercula (Tomaiuolo et al., 1999). Such dimples were infrequently observed in chimpanzee opercula, but like the dimples in the human opercula without a diagonal sulcus, these morphological features were not substantial enough to be considered sulci. The functional significance of the diagonal sulcus is still unknown, and future functional neuroimaging investigations may offer insight by studying the relationship between diagonal sulcus morphology and lateralized cognitive functions.

There was no population-based volume asymmetry of the human frontal operculum in the present study. This is not in agreement with our previous study in 50 humans (Keller et al., 2007a) but well reflects the inconsistency in the literature regarding macroscopic asymmetry of the frontal operculum across the human population (Keller et al., 2009b). Indeed, a higher proportion of the literature has revealed statistically nonsignificant asymmetry of the pars opercularis in the human brain (Wada et al., 1975; Witelson and Kigar, 1988; Tomaiuolo et al., 1999; Knaus et al., 2006, 2007; Keller et al., 2007b) compared with studies reporting statistically significant leftward asymmetry (Falzi et al., 1982; Albanese et al., 1989; Keller et al., 2007a). Given that our previous

Table 4. Volumes of the left and right pars opercularis in 10 chimpanzees in whom the inferior precentral sulcus consisted of more than one segment

\begin{tabular}{clll}
\hline Chimpanzee & Segment & Left POP Vol & Right POP Vol \\
\hline 1 & Anterior & 0.77 & 1.26 \\
21 & Posterior & 1.68 & 2.28 \\
& Anterior & 0.58 & 1.21 \\
2 & Posterior & 0.95 & 1.67 \\
& Anterior & 1.12 & 1.24 \\
& Middle & & 2.04 \\
23 & Posterior & & 2.20 \\
& Anterior & 1.24 & 1.39 \\
$\mathbf{2 6}$ & Posterior & 1.45 & 1.87 \\
& Anterior & $\mathbf{0 . 7 8}$ & $\mathbf{0 . 8 5}$ \\
$\mathbf{9}$ & Posterior & $\mathbf{1 . 2 6}$ & \\
& Anterior & $\mathbf{1 . 2 1}$ & $\mathbf{1 . 2 9}$ \\
14 & Posterior & $\mathbf{1 . 6 6}$ & \\
& Anterior & 0.50 & 1.13 \\
28 & Posterior & 0.89 & \\
& Anterior & 0.73 & 1.31 \\
& Middle & 1.69 & 2.51 \\
$\mathbf{2 0}$ & Posterior & 2.37 & \\
& Anterior & $\mathbf{0 . 5 6}$ & $\mathbf{0 . 8 2}$ \\
$\mathbf{3 0}$ & Posterior & $\mathbf{1 . 0 6}$ & \\
& Anterior & $\mathbf{1 . 0 5}$ & $\mathbf{0 . 9 9}$ \\
& Posterior & & $\mathbf{1 . 5 5}$ \\
\hline
\end{tabular}

Bold indicates the chimpanzees in whom volume asymmetry ( $\mathrm{Vol}$ ) changed direction when the posterior segment of the inferior precentral sulcus was used as the posterior boundary for the pars opercularis (POP).

study indicating leftward asymmetry of the operculum was obtained using a two-dimensional sampling method (Keller et al., 2007a), which contrasts to the null finding obtained using a three-dimensional sampling method in the present study, it may be tempting to speculate that differences in methodological approaches may account for differences in reported (a)symmetry. However, using the identical two-dimensional approach as that used by Keller et al. (2007a), we also reported no significant (or trend for) asymmetry of the frontal operculum in another sample of humans (Keller et al., 2007b). Therefore, the new threedimensional approach used in the present study cannot account for the differences in results. The real-time three-dimensional approach used in the present study permits easy separation of legitimate and illegitimate region-of-interest brain tissue, which is particularly crucial for convoluted three-dimensional structures such as gyri (Keller and Roberts, 2009). Other studies have called for measurement using all three orthogonal MR sections for anatomical specificity of other brain structures (Pruessner et al., 2000). The frontal operculum is incredibly variable in terms of sulcal morphology, and it is therefore perhaps unsurprising that this cortical region cannot be consistently asymmetric based 

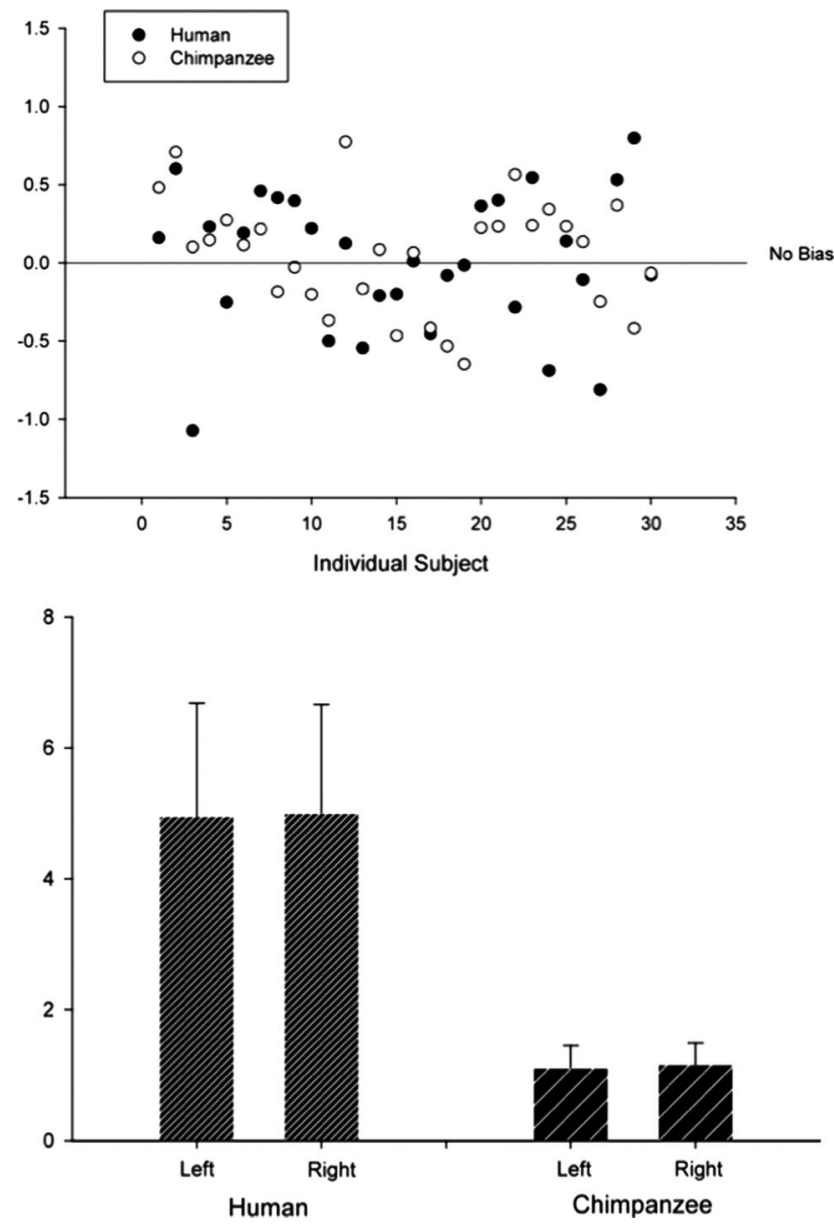

Figure 9. Results of volume estimation of the frontal operculum in humans and chimpanzees. Top, Individual volume asymmetry profiles. Negative values indicate leftward asymmetry, and positive is rightward asymmetry. Bottom, Mean volume of the left and right human and chimpanzee frontal operculum.

on quantitative measurements of gross morphology. Interhemispheric asymmetries consistently reported are generally of structures less variable in local or global structure, such as the planum temporale and fronto-occipital cerebral torque. Therefore, there is little existing evidence to suggest that gross morphological asymmetry of the frontal operculum has significance for functional lateralization in humans. Analysis of interhemispheric asymmetry and connectivity of cytoarchitectonic area 44 and area 45 , which are typically located in opercular and triangular regions of the inferior frontal gyrus, may provide insight into the lateralized organization of language in the human brain. Interhemispheric asymmetries of these regions are more consistent with functional lateralization (Keller et al., 2009b).

We did not find evidence of a left-greater-than-right volume asymmetry of the chimpanzee frontal operculum, which is in contrast to the population-based leftward asymmetry of this region reported by Cantalupo and Hopkins (2001) in a mixed sample of great apes. The divergence in results may be attributable to several factors. First, this study was concerned with cortical (gray matter) volume, whereas the previous study measured both gray and white matter within the frontal operculum. Therefore, leftward asymmetry may have been primarily determined by white matter volume in the previous study. We are currently investigating the significance of white matter anatomy in Broca's area and Broca's area homolog. Second, the present study assessed the full

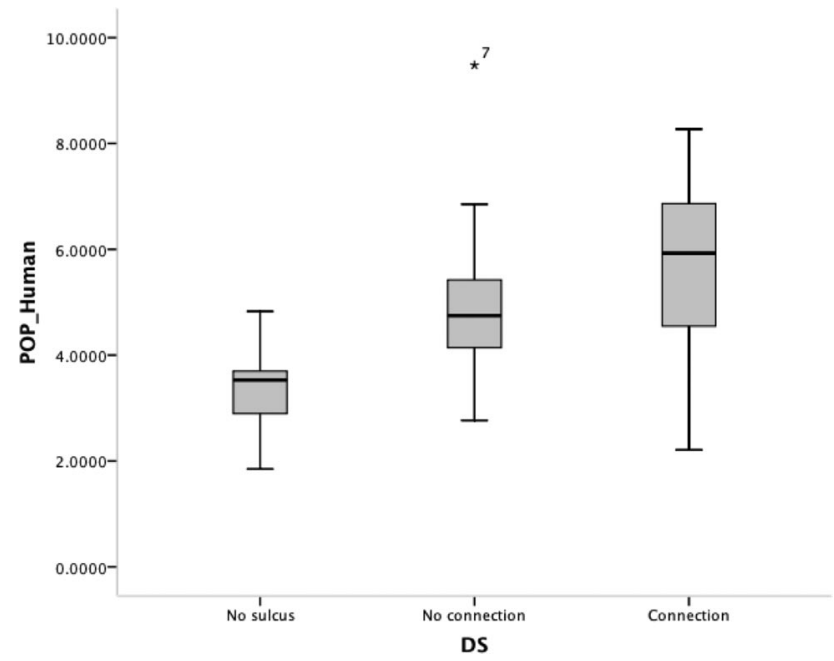

Figure 10. Box plots of the relationship between the morphology of the diagonal sulcus and volume of the frontal operculum.

extent of the intrasulcal anatomy between the fundus of sulci, whereas the previous study measured a proportion of the parasagittal area of the frontal operculum. Specifically, Cantalupo and Hopkins (2001) only quantified the gyrus lying between frontoorbital sulcus and inferior precentral sulcus and did not include any cortex above the dorsalmost point of the fronto-orbital sulcus (which is in contrast to the present study, which measured all dorsal cortex of the operculum until the fundus of the inferior frontal sulcus). Moreover, Cantalupo and Hopkins (2001) explicitly used the anterior limb of the inferior precentral sulcus as the posterior border of the operculum when inferior precentral sulcus did bifurcate. Such differences in anatomical definitions may account for differences in the reporting of asymmetry of this brain region (Keller et al., 2009b). Third, previous work has reported that asymmetry of the frontal operculum may be related to gesture handedness (Taglialatela et al., 2006). Handedness was not controlled for in the present study, and we studied a group of left- and right-handed chimpanzees, which is one possible confound. Nevertheless, whether interhemispheric asymmetry does or does not exist of the chimpanzee frontal operculum, findings need to be reconciled with the fact that asymmetry is not a robust finding in humans, most of whom are uniformly righthanded. In short, although cortical asymmetry of the operculum was not observed in the present study, it was not observed in chimpanzees or humans. Therefore, the search for the structural neuroanatomical blueprint that underlies the human capability for the acquisition and development of spoken language is unlikely to be successful in comparative studies of the gross morphology of the frontal operculum, particularly in the absence of any behavioral or functional data.

Although the results presented here suggest no asymmetry of macroscopic definitions of Broca's area and Broca's area homolog (at least that region mostly encompassing area 44), it may be that the gross morphology of the frontal operculum is not a reliable indicator of Broca's area per se. This is especially pertinent given that cytoarchitectonic boundaries are more closely related to the regional functional properties of cortex relative to sulcal landmarks, and previous studies have reported asymmetry in the cytoarchitectonic fields considered to represent Broca's area (Keller et al., 2009b), which may occasionally lie outside the frontal operculum as defined in the present study. 


\section{References}

Albanese E, Merlo A, Albanese A, Gomez E (1989) Anterior speech region. Asymmetry and weight-surface correlation. Arch Neurol 46:307-310.

Amunts K, Schleicher A, Bürgel U, Mohlberg H, Uylings HB, Zilles K (1999) Broca's region revisited: cytoarchitecture and intersubject variability. J Comp Neurol 412:319-341.

Annett M (2006) The distribution of handedness in chimpanzees: estimating right shift in Hopkins' sample. Laterality 11:101-109.

Bailey P, von Bonin G, McCulloch WS (1950) The isocortex of the chimpanzee. Urbana, IL: University of Illinois.

Barta PE, Dhingra L, Royall R, Schwartz E (1997) Improving stereological estimates for the volume of structures identified in three-dimensional arrays of spatial data. J Neurosci Methods 75:111-118.

Cantalupo C, Hopkins WD (2001) Asymmetric Broca's area in great apes. Nature 414:505.

Corballis MC (2003) From mouth to hand: gesture, speech, and the evolution of right-handedness. Behav Brain Sci 26:199-208; discussion 208-260.

Dorsaint-Pierre R, Penhune VB, Watkins KE, Neelin P, Lerch JP, Bouffard M, Zatorre RJ (2006) Asymmetries of the planum temporale and Heschl's gyrus: relationship to language lateralization. Brain 129:1164-1176.

Falzi G, Perrone P, Vignolo LA (1982) Right-left asymmetry in anterior speech region. Arch Neurol 39:239-240.

Foundas AL, Leonard CM, Gilmore RL, Fennell EB, Heilman KM (1996) Pars triangularis asymmetry and language dominance. Proc Natl Acad Sci U S A 93:719-722.

Gannon PJ, Holloway RL, Broadfield DC, Braun AR (1998) Asymmetry of chimpanzee planum temporale: humanlike pattern of Wernicke's brain language area homolog. Science 279:220-222.

Hopkins WD, Marino L (2000) Asymmetries in cerebral width in nonhuman primate brains as revealed by magnetic resonance imaging (MRI). Neuropsychologia 38:493-499.

Hopkins WD, Marino L, Rilling JK, MacGregor LA (1998) Planum temporale asymmetries in great apes as revealed by magnetic resonance imaging (MRI). Neuroreport 9:2913-2918.

Hopkins WD, Taglialatela JP, Meguerditchian A, Nir T, Schenker NM, Sherwood CC (2008) Gray matter asymmetries in chimpanzees as revealed by voxelbased morphometry. Neuroimage 42:491-497.

Keller SS, Roberts N (2009) Measurement of brain volume using MRI: software, techniques, choices and prerequisites. J Anthropol Sci 87:127-151.

Keller SS, Wieshmann UC, Mackay CE, Denby CE, Webb J, Roberts N (2002a) Voxel based morphometry of grey matter abnormalities in patients with medically intractable temporal lobe epilepsy: effects of side of seizure onset and epilepsy duration. J Neurol Neurosurg Psychiatry 73:648-655.

Keller SS, Mackay CE, Barrick TR, Wieshmann UC, Howard MA, Roberts N (2002b) Voxel-based morphometric comparison of hippocampal and extrahippocampal abnormalities in patients with left and right hippocampal atrophy. Neuroimage 16:23-31.

Keller SS, Highley JR, Garcia-Finana M, Sluming V, Rezaie R, Roberts N (2007a) Sulcal variability, stereological measurement and asymmetry of Broca's area on MR images. J Anat 211:534-555.

Keller SS, Deppe M, Roberts N, Garcia-Finana M, Ringelstein E, Knecht S (2007b) Cortical asymmetry and language lateralisation: insula and not classical language cortex predicts cerebral dominance. Neuroimage 36: S74.

Keller SS, Baker G, Downes JJ, Roberts N (2009a) Quantitative MRI of the prefrontal cortex and executive function in patients with temporal lobe epilepsy. Epilepsy Behav 15:186-195.

Keller SS, Crow T, Foundas A, Amunts K, Roberts N (2009b) Broca's area: nomenclature, anatomy, typology and asymmetry. Brain Lang 109:29-48.

Knaus TA, Bollich AM, Corey DM, Lemen LC, Foundas AL (2006) Variability in perisylvian brain anatomy in healthy adults. Brain Lang 97:219-232.

Knaus TA, Corey DM, Bollich AM, Lemen LC, Foundas AL (2007) Anatomical asymmetries of anterior perisylvian speech-language regions. Cortex 43:499-510.

Lux S, Keller S, Mackay C, Ebers G, Marshall JC, Cherkas L, Rezaie R, Roberts N, Fink GR, Gurd JM (2008) Crossed cerebral lateralization for verbal and visuo-spatial function in a pair of handedness discordant monozygotic twins: MRI and fMRI brain imaging. J Anat 212:235-248.

Ono M, Kubik S, Abernathey CD (1990) Atlas of the cerebral sulci. New York: Thieme.

Pilcher DL, Hammock EA, Hopkins WD (2001) Cerebral volumetric asymmetries in non-human primates: a magnetic resonance imaging study. Laterality 6:165-179.

Pruessner JC, Li LM, Serles W, Pruessner M, Collins DL, Kabani N, Lupien S, Evans AC (2000) Volumetry of hippocampus and amygdala with highresolution MRI and three-dimensional analysis software: minimizing the discrepancies between laboratories. Cereb Cortex 10:433-442.

Roberts N, Puddephat MJ, McNulty V (2000) The benefit of stereology for quantitative radiology. Br J Radiol 73:679-697.

Schenker NM, Hopkins W, Spocter MA, Garrison AR, Stimpson CD, Erwin JM, Hof PR, Sherwood CC (2009) Broca's area homologoue in chimpanzees (Pan troglodytes): probabilistic mapping, asymmetry and comparison to humans. Cereb Cortex. Advance online publication. Retrieved June 28, 2009. doi:10.1093/cercor/bhp138.

Sherwood CC, Broadfield DC, Holloway RL, Gannon PJ, Hof PR (2003) Variability of Broca's area homologue in African great apes: implications for language evolution. Anat Rec A Discov Mol Cell Evol Biol 271:276-285.

Taglialatela JP, Cantalupo C, Hopkins WD (2006) Gesture handedness predicts asymmetry in the chimpanzee inferior frontal gyrus. Neuroreport 17:923-927.

Tomaiuolo F, MacDonald JD, Caramanos Z, Posner G, Chiavaras M, Evans AC, Petrides M (1999) Morphology, morphometry and probability mapping of the pars opercularis of the inferior frontal gyrus: an in vivo MRI analysis. Eur J Neurosci 11:3033-3046.

Uylings HB, Jacobsen AM, Zilles K, Amunts K (2006) Left-right asymmetry in volume and number of neurons in adult Broca's area. Cortex 42:652-658.

Wada JA, Clarke R, Hamm A (1975) Cerebral hemispheric asymmetry in humans. Cortical speech zones in 100 adults and 100 infant brains. Arch Neurol 32:239-246.

Witelson SF, Kigar DL (1988) Asymmetry in brain functions follows asymmetry in anatomical form: gross, microscopic, post-mortem and imaging studies. In: Handbook of neuropsychology (Boller F, Grafman J, eds), pp 111-142. Amsterdam: Elsevier. 